Díaz de Greñu Domingo, S. \& Anguita Martínez, R. (2017). Estereotipos del profesorado en torno al género y a la orientación sexual. Revista Electrónica Interuniversitaria de Formación del Profesorado, 20(1), 219-232.

\title{
Estereotipos del profesorado en torno al género y a la orientación sexual
}

Sofía Díaz de Greñu Domingo y Rocío Anguita Martínez

Universidad de Valladolid

\section{Resumen}

En el ámbito educativo continúan detectándose numerosas muestras de sexismo y homofobia. Es fundamental descubrir y erradicar los prejuicios que el profesorado continúa transmitiendo, especialmente, a través del currículum oculto. Para completar los trabajos realizados en los años 80 y 90 y ofrecer un análisis de la realidad en la segunda década del siglo XXI se interpretan, desde una perspectiva cualitativa y crítica, los datos recopilados en un registro anecdótico y diario de observaciones durante cuatro cursos en centros de secundaria de diferentes características en Castilla y León. Los resultados revelan que los estereotipos permanecen aún arraigados pese a los esfuerzos por mostrar un clima de igualdad. La investigación en esta línea debe continuar si buscamos un sistema educativo de calidad que responda a las demandas sociales.

\section{Palabras clave}

Estereotipos de género entre el profesorado; concepciones en torno a la orientación sexual; estereotipos en el currículum oculto; discriminación sexual.

\section{Contacto:}

Sofía Díaz de Greñu Domingo

"Sofía Díaz de Greñu Domingo" sdgd@pdg.uva.es 


\title{
Teacher stereotypes around gender and sexual orientation
}

\begin{abstract}
Within the educational context numerous examples of sexism and homophobia are present. We have to eradicate prejudices which teachers continue transmitting through the hidden curriculum. In order to complete the research conducted in the $80 \mathrm{~s}$ and $90 \mathrm{~s}$ and provide an analysis of reality in the second decade of the 21st Century, data collected from an anecdotal record and systematic observations are interpreted following a critical perspective. The process takes place during four academic years in different contexts in Castilla y León. The results show that stereotypes still remain entrenched despite efforts to show a climate of equality. Research in this line must continue if we want a quality education system in line with societal demands.
\end{abstract}

\section{Key words}

Teachers' gender stereotypes; conceptions of sexual orientation; sexual discrimination; stereotypes in de hidden curriculum.

\section{Género y la orientación sexual en el sistema educativo}

El establecimiento de la actual escuela mixta en los años 70 fue un paso adelante en la lucha contra la segregación en las aulas. Sin embargo, no terminó con la discriminación entre niños y niñas en los sistemas educativos (Subirats y Brullet, 2002). Aunque esta desigualdad no se manifiesta explícitamente, aparece marcada en el currículum oculto (Gimeno, Santos, Torres y Marrero, 2015) por decisiones en los contenidos, estrategias metodológicas, relaciones interpersonales, elección de recursos didácticos, distribución del espacio y asignación de tareas (Romero, 1997; Tomé, 2001). Urruzola (1995) advertía que el avance que supusieron la Ley General de Educación y la Ley de Ordenación General del Sistema Educativo se había producido sin una crítica previa a los estereotipos de género. En los estudios de Lamb, Graling y Lustig (2011) se afirma que la nueva escuela sobrevalora los rasgos asertivos de la personalidad, vinculados a lo masculino que se relacionan con independencia, capacidad de decisión, autonomía y competitividad (Marsh, 2011). En esta línea, investigaciones recientes demuestran que la perspectiva de género debe aún abrirse paso en la formación del profesorado (Aguilar, 2013; Calvo y Rodríguez-Hoyos, 2012; Donoso-Vázquez; Montané y Pessoa, 2014).

En cuanto a las personas pertenecientes al colectivo de Lesbianas, Gays, Transexuales y Bisexuales (LGTB), se mantienen categorías negativas para ellas (Piedra de la Cuadra, Rodríguez Sánchez, Ries y Ramírez Macías, 2013) siendo necesario, que la escuela se haga cargo de la heterogeneidad. Los últimos informes de la Federación Estatal de LBTB (FELGTB) alertan de que los lugares donde más discriminación experimentan estas personas son los colegios e institutos (FELGTB, 2013a). Los signos de acoso se traducen en insultos, aislamiento y violencia física. Se denuncia también que la mayor parte del profesorado elude la cuestión y no presta la ayuda necesaria (FELGBT, 2013b).

La discriminación de las mujeres y las minorías sexuales tiene raíces y rasgos comunes. Jayme (1999) explicaba que en la ideología colectiva se identifica lo general con lo masculino y lo particular con lo femenino. Siguiendo a Kimmel (1997), la homofobia está íntimamente relacionada con el sexismo porque la homosexualidad es vista como una 
perturbación del normal desarrollo del género como constructo cultural. El heterosexismo es, según Hyde y Delamater (2006), la creencia de que todos los individuos son heterosexuales. $Y$ aunque en el ámbito educativo español no se desarrollan apenas investigaciones en esta línea, sin embargo, esto está cambiando, especialmente con la aparición de investigaciones en el mundo anglosajón (Planella y Pie, 2012).

Entre 1994 y 1999 el equipo de coeducación del Instituto de Ciencias de la Educación de la Universidad Autónoma de Barcelona puso en marcha un programa de investigación con el profesorado de Primaria y Secundaria. En el estudio se llegó a la conclusión de que se transmitían actitudes sexistas que también se describen en el trabajo de Bonal (1997). Diez años después los prejuicios del profesorado en cuanto a la perspectiva de género se mantienen (Hernández Morales, Jaramillo y Cerviño, 2005; Rebollo y García, 2009). Asimismo, el peso de los estereotipos en torno a la homosexualidad es tan fuerte que, a pesar del cuerpo normativo que surge en los últimos años para respetar el principio de no discriminación, las prácticas sociales marginan.

Por otro lado, algunos de los estudios citados describen la situación de hace diez años por lo que se debe exponer el resultado de las transformaciones en la última década. Rocha, Labraña y García Marín (2010) explican la necesidad de descubrir los estereotipos en el profesorado y Talburt y Rasmussen (2010) piensan que hay que abrir nuevas vías en la investigación analizando los puntos de partida.

\section{Origen de los estereotipos y consecuencias en la actualidad}

El paradigma estructuralista de Levi Strauss se basa en la premisa de que la mente humana organiza ideas. Se define estereotipo como una imagen entre el individuo y la realidad. En los años 20 aparece como constructo central de la Psicología y se estudia desde la vertiente emotiva, cognitiva y social. Los estereotipos establecen pautas diferenciadoras entre personas que delimitan las posibilidades individuales (Bowen, Wegmann y Webber, 2013).

Las estructuras culturales de género tienen antecedentes filosóficos e históricos. Se trata de un subtipo de estereotipos sociales que incluye lo que se espera que deben «hacer y ser» las mujeres y los hombres. Investigaciones como las de Mulac, Giles, Bradac y Palomares (2013) demuestran que esta clase de estereotipos invade nuestra forma de pensar, corroboran las tesis del feminismo clásico de Betty Friedan, Simone de Beauvoir, Judit Butler y Luce Irigaray. Generalmente contienen gran carga peyorativa de la condición femenina.

Los estereotipos de género no son estables, cambian de una sociedad a otra, puesto que se crean y varían a lo largo del espacio y del tiempo (Rocha, Labraña y García Marín, 2010). Se ha producido una evolución motivada por nuevas condiciones socioeconómicas (mayor nivel educativo y aumento del consumo que hace necesario el trabajo de la mujer).

Este modelo ideológico ha dado lugar al llamado sistema sexo-género, un esquema determinista en el que el sexo biológico condiciona destinos sociales diferentes (Archer, 2007). Se establece lo que es adecuado para hombres y mujeres reforzando categorías. «Los sistemas de género -no importa en qué periodo histórico- son sistemas binarios que oponen la hembra al macho, lo masculino a lo femenino, rara vez sobre la base de igualdad, sino, por lo general, en términos jerárquicos» (Conway, Bourque y Scott, 2000, p. 6). Las niñas asumen desde los primeros años el papel social de la feminidad hegemónica, en parte, por la influencia de los discursos del profesorado (Rodríguez y Torío, 2005). La consecuencia es una realidad marcadamente discriminatoria. El sexo es un factor determinante en la inserción laboral (Gómez Vázquez y Porto, 2010). Las mujeres presentan 
un nivel salarial 30\% menor que el de los hombres, triplicando el tiempo que emplean los varones en las tareas domésticas (Moreno, 2010; Secretaría de Mujer e Igualdad, 2015). Todavía está pendiente la incorporación de los valores del cuidado a la identidad de género masculina (Rald Philipp, 2010; Stolke, 2004). Se las excluye en puestos de liderazgo (Castillo Mayen, 2011). Ion, Durán y Bernabeu (2013) exponen que las responsabilidades familiares frenan la carrera académica de las mujeres y las políticas de igualdad no ofrecen resultados satisfactorios.

El sistema sexo-género tiene, además, una consecuencia ideológica que marca los prejuicios frente a las personas LGTB (Penna y Mateos, 2014; Pichardo, 2015). La teoría Queer como rechazo de la exclusión señala que este sistema está siempre ligado a las normas de la heterosexualidad percibida como única forma de relación sexual natural (Blaise y Taylor, 2012).

Consuelo Chacártegui (2001) recordaba que existe discriminación por razón de orientación sexual en el acceso al empleo por la suposición de falta de aptitud para ciertos puestos y en las condiciones laborales por discriminación indirecta. Ortiz-Hernández (2004) enumeraba cinco tipos de opresión de las personas LGTB: a) invisibilidad, se ignora su participación en la vida social de forma productiva, no cuentan con referentes positivos por lo que, a veces, sienten soledad y aislamiento; b) asignación de significados negativos; c) negación de oportunidades para el acceso a recursos y servicios en el ámbito laboral y médico; d) violencia verbal, física y sexual; y e) marginación en guetos por segregación.

Los estereotipos en torno al género y a la orientación sexual que han predominado conducen, pues, a la discriminación y el sistema educativo desempeña una función clave en la transmisión y reproducción de los mismos. Teniendo en cuenta lo anterior, en el presente artículo se exponen los resultados del estudio puesto en marcha durante cuatro cursos (2010/11 a 2013/14) con el objetivo de buscar e interpretar prejuicios que actualmente conserva el profesorado de enseñanza secundaria con respecto a los descritos en investigaciones de los años 80 y 90, describiendo las diferencias entre los discursos formal e informal y el modo de transmitirlos en la práctica docente cotidiana (véase tabla 3). Tras la consulta de los estudios más recientes y el análisis de las primeras observaciones se detecta que los cambios en las representaciones sobre los temas mencionados de uno de los principales agentes en el proceso de enseñanza-aprendizaje (el profesorado) no se producen tan rápidamente como sería deseable.

\section{Trabajo de campo}

Teniendo en cuenta la naturaleza de los hechos investigados, la metodología cualitativa es adecuada para lograr el objetivo propuesto, dado que se manifiesta especialmente apta para comprender los aspectos educativos en un contexto cotidiano (Angrosino, 2012).Tanto en la planificación de instrumentos de investigación como en el análisis de datos, se partió de una perspectiva interpretativa y crítica puesto que se valoraron los comportamientos y se reflexionó en torno a un tipo de dominación social. De acuerdo con la terminología usada por Tójar (2006) y Bisquerra (2012), se utilizaron las perspectivas fenomenológicas, naturalistas, interaccionistas simbólicas, críticas e interpretativas.

Entre esta heterogeneidad de paradigmas cabe destacar las perspectivas feminista y freiriana que reivindican una forma diferente de percibir el mundo y se orientan a la lucha contra la desigualdad. Rodríguez, Gil y García (1996) definen la Etnografía como método por el que se aprende el modo de vida de unidades sociales que, en este caso, son grupos de profesores y diferentes clases de varios centros educativos que se detallan en el siguiente 
apartado. Es la metodología más idónea para descubrir creencias, valores y motivaciones (Guba y Lincolm, 2002; Woods, 1987). Se emplearon herramientas etnográficas porque se perseguía la comprensión global de determinados aspectos culturales interpretando significados y funciones de las acciones humanas en contextos concretos (Guber, 2004).

\section{Registro de anécdotas y diario de observación}

Se recurrió a dos instrumentos etnográficos de primer orden: el registro de anécdotas y la observación sistemática (véase tabla 1). El proceso de investigación se llevó a cabo durante los cursos 2010/11 a 2013/14 en dos institutos de carácter rural (alrededor de 500 estudiantes matriculados procedentes de núcleos dispersos y oferta educativa modesta) y dos de carácter urbano (en torno a las 1000 matrículas y amplia oferta).

Los anecdotarios son técnicas de observación no sistemática en los que se recogen episodios significativos. No pueden ser sistemáticos porque se debe esperar el momento en que los hechos se producen (Pérez Serrano, 1994). Por esa razón, esta investigación se prolongó varios cursos con el fin de obtener una muestra suficiente de registros. Se plasmaron cronológicamente los comentarios, se describieron e interpretaron. Se optó por esta herramienta porque las anécdotas permiten revelar ideas no reconocidas en ámbitos institucionales. Se prestó atención a conversaciones, preferiblemente informales, en descansos y reuniones de coordinación del profesorado donde aparecen marcados estereotipos. Se buscó la variedad de contextos (centros descritos anteriormente) y la diversidad de perfiles del profesorado en cuanto a trayectoria profesional, edad, formación, situación laboral, participación en proyectos de innovación, etcétera (véase tabla 2), para garantizar la triangulación de tiempo, de contextos y de fuentes (Ruiz Olabuénaga, 2012).

Con el fin de valorar la forma de transmitir dichos estereotipos en la práctica cotidiana se puso en macha un diario de campo durante los dos últimos cursos en los mismos centros donde se habían recogido las anécdotas. En este caso, al tratarse de una técnica metódica, no era necesario prolongarla más en el tiempo. Una vez por semana la investigadora se incorporó a diferentes clases (mensualmente cambiaba de grupo para lograr registros de todos los niveles de la ESO y Bachillerato), solicitando la colaboración al profesorado para que impartiese sus materias con normalidad tanto en los centros rurales como urbanos.

Para realizar este diario se empleó un protocolo, elaborado a partir de estudios previos sobre actitudes del profesorado en cuestiones de género (Hidalgo, Juliano, Roset y (aba,2003), que incorporaba nuevos elementos relacionados tanto con prejuicios sexistas como de orientación sexual, a saber: frecuencia de intercambios con chicos y chicas, tono empleado con unos y otras, alusión a la desigualdad de género y a la diversidad LGTB, desarrollo de actividades para promover la no discriminación y estereotipos detectados.

Tabla 1.

Instrumentos de recogida de datos

\begin{tabular}{|l|l|l|l|}
\hline Instrumentos & Personas implicadas & Contexto & Periodicidad \\
\hline Registro anecdótico & Profesorado diverso & Salas reunión & $2010 / 11$ a 13/14 \\
\hline Diario de campo & Ídem & Aulas de clase & $2012 / 13$ y 13/14 \\
\hline
\end{tabular}


Durante la investigación se estableció un criterio de respeto y compromiso a largo plazo (Angrosino, 2012). Para ello, una vez recogidas las anécdotas, se informó de los resultados a los centros y se explicó cómo iban a ser utilizados garantizando la confidencialidad. Se respetaron los principios de privacidad, dignidad, intimidad y sensibilidad (Angrosino, 2012; Pérez Serrano, 1994; Tójar, 2006). En el curso del proceso se persiguieron los principios de credibilidad. Se recogieron datos de forma lenta, prolongada y sistemática, buscando la riqueza de contenidos y comprobando por medio de la triangulación de líneas teóricas, de fuentes, de personas y de instrumentos la coherencia (Guba y Lincolm, 2002). El trabajo se revisó desde el ámbito universitario para corregir los posibles sesgos de la observadorainvestigadora y garantizar la confirmabilidad (Gómez, Latorre, Sánchez y Flecha, 2006).

Tabla 2.

Proceso de triangulación

\begin{tabular}{|l|l|l|l|l|}
\hline \multicolumn{2}{|l|}{ Triangulación } \\
$\begin{array}{l}\text { Presupuestos } \\
\text { teóricos }\end{array}$ & $\begin{array}{l}\text { Personas } \\
\text { participantes }\end{array}$ & Tiempo & $\begin{array}{l}\text { Contexto y } \\
\text { fuentes }\end{array}$ & Instrumentos \\
\hline
\end{tabular}

A lo largo del proceso, se detectaron numerosos comentarios estereotipados. Dichos comentarios repetían los mismos patrones en centros, situaciones y momentos diferentes. Se llevó a cabo un proceso de clasificación siguiendo el criterio marcado en los objetivos.

Tabla 3.

Categorías

\begin{tabular}{|l|l|}
\hline Categorías & Instrumentos \\
\hline Estereotipos género y diversidad sexual & Registro de anécdotas y diario \\
\hline Evolución del pensamiento & Ídem \\
\hline Práctica en el aula & Diario de observación \\
\hline
\end{tabular}

\section{Estereotipos de género en el imaginario del profesorado}

Las investigaciones realizadas de hace más de una década (Ayala y Mateo, 2005) ponían de manifiesto que, aunque el profesorado negaba poseer sesgos de género, se apreciaban diferencias de trato, se hacían bromas sexistas, se producían comentarios basados en estereotipos, se adjudicaban determinadas tareas dependiendo del sexo, había negligencia hacia ciertos comportamientos agresivos y se fomentaba la competitividad en los chicos y la pasividad en las chicas.

Deaux y Lewis (1984, citado por Jayme, 1999) establecían tres grandes categorías en cuanto a los estereotipos de género: referidos a rasgos de la personalidad, ligados a las funciones tradicionalmente asignadas a hombres y a mujeres y a la apariencia física con raíces en el dimorfismo sexual. Características como asertividad, independencia y autonomía se ligaban a lo masculino, mientras que sensibilidad, emotividad y afectividad a lo femenino (Piusi, 2001). Estas categorías siguen manteniéndose en la actualidad (Cubero et al., 2015). Los 
resultados de las observaciones y del registro de anécdotas pusieron de manifiesto que se mantienen estereotipos que responden a las taxonomías de los estudios anteriores.

La clasificación que se expone a continuación está basada en comentarios recogidos a lo largo de cuatro años. Siguiendo las recomendaciones de Guba y Lincolm (2002), en la interpretación se utilizan ejemplos representativos extraídos del registro anecdótico y, en menor medida, del diario de observaciones dado que en las aulas no se produjeron, apenas, referencias explícitas.

Respecto a la capacidad de gestión, aptitud intelectual y habilidades cognitivas, Diana Halpern (2011) critica interpretaciones que, basándose en las supuestas habilidades cognitivas de hombres y mujeres, obstaculizan las aspiraciones de las mujeres. Esta afirmación se ve corroborada en las reuniones docentes observadas donde se manifiestan comportamientos que denotan diferente actitud ante unos y otras. A las profesoras se las escucha durante menos tiempo incluso aunque ejerzan la presidencia (tutoras en las juntas de evaluación, psicólogas en horas dedicadas a planificación de tutorías, jefas de estudio en comisiones de convivencia, etcétera). Los profesores protagonizan el $80 \%$ de las intervenciones. Cuando en la sala el profesorado masculino está en minoría, las profesoras mantienen debates y los hombres no suelen participar. Sin embargo, buscan conversaciones que consideran «más elevadas desde el punto de vista intelectual» con sus compañeros.

A lo largo del Claustro las opiniones de varios profesores se suceden, nadie interrumpe, a pesar de que algunos de ellos desvían la atención de los puntos del orden del día. Se acaba el tiempo y la responsable de actividades extraescolares debe informar sobre los viajes de fin de curso. Lo hace rápidamente solicitando gestualmente la aprobación de sus compañeros. Alguien enseguida le arrebata el turno de palabra. (Registro anecdótico, 2014)

Rocha, Labraña y García Marín (2010) señalan que el profesorado tiene unas expectativas diferentes en cuanto a capacidades. Se cree, aunque no se reconoce abiertamente, que las mujeres necesitan compensar sus dificultades intelectuales con tesón. Cualidad que raramente se atribuye a los hombres: «Pobrecita es muy trabajadora pero le cuesta mucho comprender», comenta el jefe de estudios en una sesión de evaluación de $3^{\circ}$ de ESO (Registro anecdótico, 2012).

La devaluación de la intuición en los métodos científicos tradicionales ha sido asociada con características femeninas (Martín Casares, 2006). Se adjudican a los hombres en exclusiva algunas destrezas como la lógica o la orientación espacial: "Una profesora explica que, después de dos años en el centro, continúa perdiéndose por las instalaciones: "Menos mal que me acompañas" dice a un compañero». (Registro anecdótico, 2012).

Hay una desconfianza ante la aptitud de las mujeres ante el manejo de las Tecnologías de la Información y la Comunicación (TIC). Normalmente se recurre a un hombre cuando surgen problemas técnicos: «En clase la profesora tiene problemas al iniciar el ordenador. Pide ayuda a uno de los alumnos en un grupo donde constituyen minoría» (Diario de observación, 2013).

En relación a las características psicológicas y físicas, Verena Stolke (2004) recordaba la incapacidad de ninguna investigación para demostrar que existan diferencias intelectualesemocionales innatas relevantes en hombres y mujeres. El sentimentalismo y la emotividad se suponen caracteres femeninos que se oponen a la racionalidad presuntamente masculina: «En la sala se oye decir sobre una alumna: "Está bajando su rendimiento, ¡claro! Ya comienzan a tontear con los chicos". Esta observación se ha repetido en varias reuniones de evaluación» (Registro anecdótico, 2011). 
Es frecuente identificar el carácter arisco a mujeres solteras y a todas, en general, a partir de una cierta edad: “"¡Qué carácter tiene! ¡A ver si se busca un novio y se le pasa!” dice una profesora a una compañera». (Registro anecdótico, 2012). "Dos compañeros charlan con otro que acaba de tener un hijo "Ahora tendrás todos los días a la suegra en casa" le dicen con sarcasmo». (Registro anecdótico, 2012). Por otra parte, a las mujeres se les presupone cierto carácter maligno. "“QQué miedo me dan las mujeres!" dice un profesor a otro con tono afable». (Registro anecdótico, 2011). También aparece habitualmente el estereotipo de la mujer cotilla:

Varias profesoras comentan a la hora del recreo las últimas noticias de las revistas del corazón. Están cansadas y necesitan bromear. Entra un compañero y al verlas afirma: « ¡las mujeres siempre criticando!» Al cabo de un rato la observadora es testigo de una conversación entre dos profesores sobre la vida personal de otro compañero. (Registro anecdótico, 2014)

Se piensa que las mujeres deben preocuparse en mayor medida por su apariencia física: "“QQué lástima que una chica vaya tan desarreglada!”, comenta una profesora». (Registro anecdótico, 2010), y se asocian los problemas de salud a la función reproductora: «Una profesora tiene calor y abre las ventanas. Un compañero se divierte hablando de los síntomas de la menopausia» (Registro anecdótico, 2014).

En cuanto a la función que las mujeres deben desempeñar en la sociedad, el profesorado mantiene la idea de que las principales tareas que les corresponden son el cuidado de la familia y el hogar. En el caso de las mujeres solteras, la atención a personas mayores sobrinos, niños de personas allegadas: "Varios docentes toman café en un descanso. Uno de los profesores pregunta a un compañero: “Se casó tu hermana?" "No", responde. "Es mejor así, estoy tranquilo porque se ocupa de mis padres", añade» (Registro anecdótico, 2011). La posibilidad de dar prioridad a su vida profesional o a otro tipo de actividades al margen del cuidado del hogar no se tiene en cuenta.

Ciertas cualidades como el orden, la prudencia, la disciplina, etcétera, se asocian a lo femenino: “"Si no fuera por las mujeres, ¡cómo estarían las casas!", afirma una profesora». (Registro anecdótico, 2010). «Al pasar frente a una de las clases se puede ver a la tutora diciendo a varias alumnas: "Parece mentira que unas chicas tengan todo tan desordenado"» (Registro anecdótico, 2011).

Cuando se refieren al fenómeno de la doble jornada de las mujeres no se plantean un cambio. "Mi marido no hace nada de la casa y yo lo prefiero así. Es un desastre", se oye comentar en una conversación». (Registro de anécdotas, 2012), ““iQué cuaderno tan desordenado! Los chicos son muy 'chapuceros', es una frase recurrente». (Registro de anécdotas, 2010). Por el contrario, suponen que la agresividad en los chicos es una característica innata que les permitirá en el futuro defenderse y proteger a su familia ante las amenazas:

El jefe de estudios entra en el aula, dos chicos se han peleado y quiere advertir al grupo que ese tipo de conductas no debe repetirse. Al entrar en clase se da cuenta de que solo hay alumnas por lo que expone: «bueno, aunque normalmente las chicas no os peleáis, no quiero que se repita esto. Transmitídselo a vuestros compañeros». (Registro de anécdotas, 2014)

Una profesora acompaña a una alumna que ha roto una persiana de la clase a jefatura. Normalmente ante este tipo de actos se exigen responsabilidades. En este caso, el jefe de estudios afirma que probablemente la persiana no estuviera en buen estado para justificar la acción de la adolescente». (Registro de anécdotas, 2011)

Existe la creencia mayoritaria de que la desigualdad se ha superado en el ámbito educativo. Se califican como discriminatorias para los hombres las medidas de acción positiva. "La profesora de Ciencias Sociales señala: "Todo el mundo debe partir del mismo lugar. Las 
mujeres no deben ocupar altos cargos si tienen menos méritos"» (Registro de anécdotas, 2010). También es habitual que no se reconozca explícitamente la existencia de discriminación:

El día 8 de marzo un profesor exclama en tono jocoso: “iNo sé de qué os quejáis las mujeres! En algunas empresas privadas existe la discriminación laboral pero no entre los funcionarios"». (Registro de anécdotas, 2012). "En este instituto organizamos variadas actividades contra la violencia de género y se realizan programas de educación afectivosexual", justifica un compañero. (Registro de anécdotas, 2013)

De las observaciones en las aulas se ha podido colegir, teniendo en cuenta el protocolo y categorías empleados en esta investigación, que la frecuencia de interacción en las aulas es mayor con los chicos, el tono empleado con ellos denota mayor vehemencia, a nivel formal hay una contención a la hora de emitir ciertos comentarios por lo que los estereotipos son difíciles de detectar en este contexto. Con el paso del tiempo esta contención aumenta pero no va acompañada de programación de actividades que aborden sistemáticamente la igualdad.

\section{La invisibilidad de la diversidad afectivo-sexual}

Con respecto a la diversidad afectivo-sexual todavía se mantienen hoy prejuicios heredados. Las teorías homófobas han relacionado homosexualidad a civilizaciones decadentes y a delitos sexuales, partiendo de que esta variedad puede aparecer o desaparecer dependiendo de las circunstancias y es antinatural. Por otro lado, aunque buena parte de la sociedad ha superado estas ideas al poseer mayor cantidad de información, algunas personas creen que la orientación sexual puede modificarse pero, paradójicamente, no lo aplican a la heterosexualidad. Otros mitos corrientes asocian homosexualidad a promiscuidad, extravagancia, incapacidad de sentir amor y, por tanto, no discriminación a la hora de elegir pareja sexual (Penna y Mateos, 2014, Pichardo, 2015).

La transgresión de ámbitos está especialmente castigada en los chicos que tienen que aprender a reprimir sus sentimientos y rechazar todo aquello que se identifique con su parte femenina (Jayme, 1999). El hombre homosexual, según señalaba Ortiz-Hernández (2004), renuncia al poder que otorga la masculinidad y por eso es sancionado socialmente. La homosexualidad sigue sin aceptarse: «Dos profesoras recuerdan a un alumno al que conocieron en clase hace dos cursos. Una de ellas dice: "me he enterado de que sale con un chico. No puedo creer que sea gay". "Yo ya lo veía algo extraño" contesta». (Registro de anécdotas, 2012). "'Los chicos le llaman 'marica" dice una profesora a otra. "Es que mira que es rarito el chico", contesta» (Registro de anécdotas, 2010).

La información que existe actualmente no impide que la mayoría del profesorado, al igual que sucede fuera del ámbito educativo, suponga heterosexualidad en todas las personas: "Varios adolescentes en el recreo se burlan de un compañero porque es amanerado. Una profesora comenta: "que sea amanerado no quiere decir que sea homosexual"» (Registro anecdótico, 2011).

Un porcentaje importante piensa que se trata de una situación pasajera elegida libremente de forma caprichosa, lo cual supone un gran retroceso porque ya en el siglo XIX, aunque se consideraba algo patológico, se eximía a la persona de "responsabilidad»: "Hay que respetar las opciones sexuales" comenta una profesora, "y más ahora que se ha puesto de moda", dice su compañera». (Registro anecdótico, 2011). Esta idea se relaciona con la asociación de homosexualidad a ciertas conductas inmorales reduciendo la persona a su condición sexual: «En una reunión de tutores, un profesor, hablando de las miradas que 
algunos alumnos le dirigen cuando los descubre fumando, exclama " $i$ esto es como en Chueca! ¡Vaya insinuaciones!” (Registro anecdótico, 2010).

Aún en nuestros días la exhibición del amor homosexual no es tolerada de la misma forma que la de las relaciones heterosexuales: "“Cuando voy a Madrid me quedo extrañado al ver a los gais", dice una profesora joven». (Registro anecdótico, 2009). La bisexualidad no es bien aceptada ni comprendida. Se considera una excusa para ocultar la perversión y el libertinaje. Se ridiculiza. «"Le han pillado en una orgía con hombres y mujeres. "¡Qué suerte! ¡Hay que probar de todo!", bromea una profesora» (Registro de anécdotas, 2010).

Se atribuye a los hombres homosexuales ciertas características relacionadas con la mayor sensibilidad, sentido estético y refinamiento y a las mujeres no heterosexuales mayor independencia y autonomía. El sistema sexo-género está presente en las representaciones: «"Estos gais ¡qué bien visten!", comenta un profesor en la sala mientras ojea un periódico». (Registro de anécdotas, 2012). "“Los chicos no se atreven a enfrentarse con las lesbianas, tienen miedo" bromea un profesor mientras pasea por el patio» (Registro de anécdotas, 2011).

La familia perfecta es concebida siguiendo un modelo ideal. Se utiliza, a menudo, el término «familia desestructurada» para aquélla que se aparta de este esquema. Existen numerosos prejuicios hacia las familias monoparentales y reconstituidas y no se alude a las familias homoparentales:

En el transcurso de una reunión de tutoría la orientadora comenta algunos aspectos de la situación personal de un alumno. «"Hay que tener en cuenta la situación familiar. El padre se marchó. Vive con la madre. La madre está desbordada». (Registro anecdótico, 2010)

Después de diez años tras haber aprobado el matrimonio entre parejas del mismo sexo, este contrato constituye, junto con el derecho a la adopción que lleva aparejado, un tema controvertido:

Durante una reunión de tutoría se nos informa sobre las circunstancias de una niña adoptada por una pareja de dos mujeres. " ¿No provocará esto rechazo en el grupo?» dice una profesora. La orientadora explica que hasta el momento no se ha detectado ningún problema. (Registro de anécdotas, 2012)

Ante las circunstancias descritas, es lógico deducir que la diversidad afectivo-sexual no se tiene en cuenta como contenido en las diferentes materias del currículum: " ¡A saber qué pensarían los padres si abordamos este tema en las clases!», señala un profesor cuando una de las investigadora le explica que está programando unas actividades al respecto». (Registro de anécdotas, 2010). Se piensa que no es cuestión que haya que tratar en la esfera pública y, sin embargo, el sistema debe garantizar el bienestar del conjunto del alumnado y, por tanto, estudiar y comprender este tipo de diversidad. Se elude la responsabilidad:

La investigadora comenta en una reunión en el centro de formación que debería haber más cursos sobre la discriminación por orientación sexual en los centros educativos. Eso es algo que deben aprender fuera de la escuela, afirma un compañero». (Registro anecdótico, 2011). «Dos alumnos discuten en los pasillos: “eres una 'nenaza' dice uno de ellos con tono agresivo. Los profesores no intervienen». (Registro anecdótico, 2011)

Las observaciones en el aula muestran la omisión de la cuestión LGTB en las clases, incluso cuando se emiten comentarios al respecto por adolescentes. La homosexualidad femenina se ignora también en el ámbito no formal (Penna y Mateo, 2014). No se hallan apenas anécdotas en este sentido. Durante el periodo observado no se puso en marcha actividad alguna que abordase lo anteriormente mencionado. 


\section{Reflexiones finales}

A pesar de las limitaciones del estudio (necesidad de continuidad por los rápidos cambios sociales nuestros días, complementación con otros trabajos que conduzcan a una mayor generalización de los resultados), se ha constatado, siguiendo las categorías establecidas, que apenas hay variaciones en la forma de pensar del profesorado en la actualidad con respecto a finales del siglo XX. Perduran prejuicios asociados al sistema sexo-género: diferente capacidad intelectual y rasgos de la personalidad que se ligan al sexo biológico. Por otra parte, el grupo docente, en líneas generales, no acepta la orientación no heterosexual con la naturalidad que se aprecia en las nuevas generaciones (Serrano, Gómez, Amat y López, 2012).

La diferencia con respecto a épocas pasadas estriba, según ponen de manifiesto las observaciones, en que se pone mayor empeño en disimular estas representaciones mentales porque las manifestaciones explícitas de sexismo y homofobia no se toleran socialmente. Se hacen mayores las diferencias entre los discursos formal e informal. Se omiten ciertos temas considerados controvertidos como se ha demostrado a lo largo de esta investigación dado que puede ser motivo de rechazo lo que no significa un verdadero cambio en profundidad en el modo de pensar.

Aunque, en ocasiones, se desarrollan actividades en pro de la igualdad en días concretos, estas experiencias aisladas no tienen apenas repercusión en el proceso formativo. Como elementos positivos, se detecta que los comentarios estereotipados disminuyen en los últimos años y que la participación en proyectos de igualdad no es rechazada abiertamente como en el caso de la colaboración solicitada en este estudio. Queda aún un largo camino por recorrer hasta que se produzca la concienciación de que el sistema educativo debe hacer mayor hincapié para corregir las desigualdades también en este terreno. Por todo ello, el profesorado debe reflexionar sobre sus concepciones y abordar ciertas cuestiones en la marcha cotidiana de las clases.

\section{Referencias}

Aguilar Ródenas, C. (2013). Género y formación crítica del profesorado: una tarea urgente y pendiente. Revista Interuniversitaria de Formación del Profesorado, 78 (27,3), 177-185.

Angrosino, M. (2012). Etnografía y observación participante en Investigación Cualitativa. Madrid: Morata.

Archer, L. (2007). Class, gender, (hetero) sexuality and schooling: paradoxes within working class girls, engagement with education and post-16 aspirations. British Journal of Sociology of Education, 28(2), 165-180.

Ayala, A.I. y Mateo, P. L. (2005) (Coord.). Educar en relación, estereotipos y conflictos de género. Zaragoza: Departamento de Educación y Ciencia del gobierno de Aragón.

Bisquerra Alzina, R. (Coord.) (2012). Metodología de la investigación educative. Madrid: La Muralla.

Blaise, M. y Taylor, A. (2012). Using Queer Theory to Rethink Gender Equity in Early Childhood Education. Young Children, 67 (1), 88-96.

Bonal, X. (1997). Las actitudes del profesorado ante la coeducación. Propuestas de intervención. Barcelona: Graó. 
Bowen, N. K., Wegmann, K. M., Webber \& Kristina C. (2013). Enchancing a Brief Writing Intervention to Combat Stereotype Threat among Middle-School Students. Journal of Educational Psychology, 105 (2), 427-435.

Calvo Salvador, A. y Rodríguez-Hoyos, C. (2012). Aportaciones de los estudios de las mujeres y del género a la organización escolar. Estado de la cuestión en España. Educación $X X I, 15$ (1), 43-60.

Castillo Mayén, M. R. (2011). Discriminación de género y dominación social. Análisis de los estereotipos de género y de la influencia del priming subliminal. Tesis doctoral. Universidad de Jaén.

Cubero, M., Santamaría, A, Rebollo, M.A., Cubero, R., García, R. y Vega, L. (2015). Teacher negociating discourses of gender (in)equality: the case of equal opportunities reform in Andalusia. Gender and Education, september, 1-34 doi: 101080/09540253.2015.1083947.

Chacártegui, C. (2001) (1 $1^{\mathrm{a}}$ ed.). Discriminación y orientación sexual del trabajador. Valladolid: Editorial Lex Nova.

Conway, J. K., Bourque, S. C. y Scott J. W. (2000). El concepto de género. En M. Lamas (Comp.), El género. La construcción cultural de la diferencia sexual, 227-238. México: Grupo Editorial Miguel Ángel Porría.

Donoso-Vázquez, T., Montané, A. y Pessoa, M.E. (2014). Género y calidad en educación superior. Revista Electrónica Interuniversitaria de Formación del Profesorado. 17(3), 114.

Federación Estatal de Lesbianas, Gays, Transexuales y Bisexuales (2013a). Estudio sobre discriminación por orientación sexual y/o identidad de género en España. Recuperado de http://www.felgb.org.

Federación Estatal de Lesbianas, Gays, Transexuales y Bisexuales (2013b). Acoso escolar (y riesgo de suicidio) por orientación sexual e identidad de género: Fracaso del Sistema Educativo. Recuperado de http://www.felgb.org.

Gimeno Sacristán, J., Santos Guerra, M.A., Torres Santomé, J., Jackson, P. W. y Marrero Acosta, J. (2015). Ensayos sobre el currículum. Teoría y práctica. Madrid: Morata.

Gómez Vázquez, M. B. y Porto, A. M. (2010). Educación Superior y Mercado de Trabajo: Aspectos diferenciales en función del género. En R. M. Rald Philipp (Coord.), Investigaciones actuales de las mujeres y del género (pp. 201-216). Santiago de Compostela: Servicio de publicaciones de la Universidad.

Gómez, J., Latorre, A., Sánchez, M., y Flecha, R. (2006). Metodología comunicativa crítica. Barcelona: El Roure.

Guba, E. G. y Lincoln, Y. S. (2002). Paradigmas en competencia en la investigación cualitativa. En C. Denman y J. A. Haro (Comps.), Por los rincones. Antología de métodos cualitativos en la investigación social. El colegio de Sonora (pp.113-145). Sonora: Hermosillo.

Guber, R. (2004) (2 $2^{\mathrm{a}}$ ed.). La etnografía, método, campo y reflexividad. Bogotá: Editorial Norma.

Halpern, D. F. (2011). Sex Differences in Cognitive Abilities. Fourth Edition. Florence: Psychology Press, Taylor \& Francis Group. 
Hyde, J. y Delamater, J. D. (2006). Understanding human sexuality (9th ed.). Boston (USA): McGraw Hill.

Hernández Morales, G., Jaramillo, C. y Cerviño, M. J. (2005). Tomar en serio a las niñas. Cuadernos de educación no sexista, 17, 5-85. Recuperado de http://www.inmujer.gob.es/areasTematicas/educacion/publicaciones/serieEducacNo Sexista/docs/cuaderno17.pdf

Hidalgo, E., Juliano, D., Roset, M., y Caba, A. (2003). Repensar la enseñanza de la geografía y la historia. Una mirada desde el género. Barcelona: Octaedro.

Ion, G., Durán, M. M. y Bernabeu, M. D. (2012). El profesorado y su percepción sobre la igualdad de género en la Universidad. Revista Complutense de Educación, 24 (1), 123140.

Jayme, M. (1999). La identidad de género. Revista de Psicoterapia, Vol. X, 40, 5-22.

Kimmel, M. S. (1997). Homofobia, temor, vergüenza y silencio en la identidad masculina. Isis Internacional. Ediciones de las mujeres, 24, 49-62.

Lamb, S., Graling, K. \& Lustig, K. (2011). Stereotipes in Four Current AOUM Sexuality Education Curricula: Good Girls, Good Boys, and the New Gender Equality. American Journal of Sexuality Education 6 (4), 360-380.

Marsh, V. (17 de septiembre de 2011). Los estereotipos afectan al rendimiento académico de las mujeres. Tendencias sociales.

Martín Casares, A. (2006) (1 $1^{\mathrm{a}}$ edición). Antropología del género. Culturas, mitos y estereotipos sexuales. Madrid: Cátedra.

Moreno, A. (2010). Mujeres y Ciencia: de la negación y la invisibilidad a las aportaciones para la renovación de las Humanidades y las Ciencias Sociales en la era digital. En R. M. Rald Philipp (Coord.), Investigaciones actuales de las mujeres y del género (pp. 239252). Santiago de Compostela: Servicio de publicaciones de la Universidad.

Mulac, A., Giles, H., Bradac, J. J. y Palomares, N. A. (2013). The Gender-Linked Language Effect: An Empirical Test of a General process Model. Language Sciencies, 38, 22-31.

Neto, A., Cid, M., Pomar, C., Peças, A., Chaleta, E. y Folque, A. (1999). Estereotipos de género. Lisboa: Comision para la Igualdad y los Derechos de las Mujeres.

Ortiz Hernández L. (2004). La opresión de las minorías sexuales desde la inequidad de género. Política y Cultura, 22, 161-182.

Penna Tosso, M. y Mateos Casado, C. (2014). Los niveles de homofobia en los futuros docentes. Revista iberoamericana de Educación, 66, 23-142.

Pichardo Galán, J.I. (Coord.) (2015). Abrazar la diversidad. Instituto de la Mujer y para la Igualdad de Oportunidades.

Planella, J. y Pie, A. (2012). Pedagoqueer: resistencias y subversiones educativas. Educación $X X I, 15$ (1), 265-283.

Pérez Serrano, G. (1994). Investigación cualitativa. Retos e interrogantes (tomo 2). Técnicas y análisis de datos. Madrid: La Muralla.

Piedra de la Cuadra, J., Rodríguez Sánchez, A.R., Ries, F. y Ramírez Macías, G. (2013). Homofobia, heterosexismo y educación física: percepciones del alumnado. Revista de currículum y formación del profesorado 17 (1), 325-338. 
Piusi, A. M. (2001). Dar clase: el corte de la diferencia sexual. En N. Blanco (Coord.), Educar en femenino y en masculino (pp.145-165). Madrid: Akal.

Rald Philipp, R. M. (2010). Diferencias de género, empleo de las mujeres y el nuevo rol de género femenino. En R. M. Rald Philipp (Coord.), Investigaciones actuales de las mujeres y del género (pp.91-107). Santiago de Compostela: Servicio de publicaciones de la Universidad.

Rebollo, M. A. y García, R. (2009). Diagnóstico de la cultura de género en educación: actitudes del profesorado hacia la igualdad. Revista de Educación, 355, 521-546.

Rocha, T. C.; Labraña, P. A. y García, J. (2010). Expectativas del profesorado en relación al género cuando evalúan las capacidades metacognitivas de sus alumno(a)s. En R. M. Rald Philipp (Coord.), Investigaciones actuales de las mujeres y del género (pp.217228). Santiago de Compostela: Servicio de publicaciones de la Universidad.

Rodríguez Gómez, G., Gil Flores, J. y García Jiménez, E. (1999). Metodología de la investigación cualitativa. Málaga: Aljibe.

Rodríguez Menéndez, M. C. y Torío López, S. (2005). El discurso de género del profesorado de Educación Infantil: hablando acerca de la ética del cuidado. Revista Complutense de Educación, 16 (2), 471-487.

Romero, I. (1997). La coeducación como tema transversal del currículo. En M. T. Alario, C. García Colmenares y C. Alario (Coord.), Persona, Género y Educación (pp.13-21). Salamanca: Amarú.

Ruiz Olabuénaga, J. I. (2012) ( 5 a edición). Metodología de la investigación cualitativa. Bilbao: Universidad de Deusto.

Secretaría Mujer e Igualdad en Castilla y León de Comisiones Obreras (2015). Las mujeres en Castilla y León. Acercamiento a su situación social y laboral. Recuperado de http://www.castillayleon.ccoo.es/webcastillayleon/Areas:Estudios.

Serrano, F. J., Gómez García, A., Amat Muñoz, L. Ma y López Gomix, A. (2012). Aproximación a la homofobia desde la perspectiva de los estudiantes de Educación Sexual de la Universidad de Murcia. Tejuelo. Didáctica de la Lengua y la Literatura. Monográfico, 6, 74-88.

Soriano, S. (1999). Cómo se vive la homosexualidad y el lesbianismo. Salamanca: Amarú.

Stolke, V. (2004). La mujer es puro cuento. La cultura de género. Revista de Estudios Feministas, 12 (2), 1-17.

Subirats, M., Brullet, C. (2002). Rosa y azul: la transmisión de los géneros en la escuela mixta. En: González, A., Lomas, C. [Coords.]. Mujer y educación. Educar para la igualdad, educar desde la diferencia. Barcelona: Graó, pp. 133-167.

Talburt, S. y Rasmussen, M. L. (2010). "After-Queer" Tendencies in Queer Research. International Journal of Qualitative Studies in Education, 23 (1), 1-14.

Tójar, J. C. (2006). Investigación cualitativa. Comprender y actuar. Madrid: La Muralla.

Tomé, A. (2001). La construcción de las identidades masculinas y femeninas en la escuela. En N. Blanco (Coord.), Educar en femenino y en masculino 87-98. Madrid: Akal.

Urruzola, M. J. (1995). Introducción a la filosofía coeducadora. Bilbao: Maite Canal.

Woods, P. (1987). La Escuela por dentro. Etnografía de la investigación educativa. Barcelona: Paidos-MEC. 